\title{
Editorial: Tunable and Reconfigurable Optical Metamaterials
}

\author{
Yuancheng Fan ${ }^{1 *}$, Qian Zhao ${ }^{2 *}$, Fuli Zhang ${ }^{1 *}$ and Nian-Hai Shen ${ }^{3 *}$ \\ ${ }^{1}$ Key Laboratory of Light-Field Manipulation and Information Acquisition, Ministry of Industry and Information Technology and \\ School of Physical Science and Technology, Northwestern Polytechnical University, Xi'an, China, ${ }^{2}$ Department of Mechanical \\ Engineering, State Key Laboratory of Tribology, Tsinghua University, Beijing, China, ${ }^{3}$ Ames Laboratory and Department of \\ Physics and Astronomy, lowa State University, Ames, IA, United States
}

Keywords: metasurfaces, metamaterials, liquid crystals, tunable metamaterial, reconfigurable metamaterial

Editorial on the Research Topic

Tunable and Reconfigurable Optical Metamaterials

Metamaterials and 2D metasurfaces [1-8] show promising and novel methods for the manipulation of optical waves in the terahertz, infrared, and visible regimes. Their performance has been demonstrated in high-resolution imaging, nonlinear optics, radiation control, holography, and optical communications. However, their practical applications are limited by the narrow operation wavelength range resulting from the resonant nature of the constitutive microstructures.

Materials with changeable properties or reconfigurable structures are being incorporated to

\section{OPEN ACCESS}

Edited and reviewed by: Lorenzo Pavesi,

University of Trento, Trento, Italy

*Correspondence:

Yuancheng Fan

phyfan@nwpu.edu.cn

Qian Zhao

zhaoqian@tsinghua.edu.cn

Fuli Zhang

fuli.zhang@nwpu.edu.cn Nian-Hai Shen

nhshen@ameslab.gov

Specialty section:

This article was submitted to Optics and Photonics, a section of the journal

Frontiers in Physics

Received: 24 May 2021 Accepted: 28 May 2021 Published: 09 July 2021

Citation:

Fan $Y$, Zhao Q, Zhang F and Shen N-H (2021) Editorial: Tunable and Reconfigurable Optical Metamaterials. Front. Phys. 9:713966. doi: 10.3389/fphy.2021.713966 achieve tunable optical properties, i.e. to extend the operation bandwidth or parameter space of metamaterials [9-14]. For example, graphene and related 2D materials, semiconductors, phase changing materials like $\mathrm{VO}_{2}$ and $\mathrm{Ge}_{2} \mathrm{Sb}_{2} \mathrm{Te}_{5}$, liquid crystals, and MEMS-structured metamaterials are emerging for advanced optics and photonics spanning from terahertz to visible frequencies. These developments are important for both fundamental optical physics and possible applications in nonlinear nanophotonics and super-resolution imaging.

This research topic on Tunable Metamaterial/Metasurface includes some remarkable examples. From the theoretical side, Xu et al. reported a study on the tunable optical scattering properties of a kind of plasmonic nanoantenna which is composed of metal-dielectric-metal metamaterial embedded with PTsymmetric layers designed for unidirectional scattering functionality. Yang et al. exploited the radiation characteristics of an ultrathin Pt layer and impedance matching to design a wavelength-selective absorber based on planarized platinum/silicon (Pt/Si) multilayer film for infrared stealth. The absorber effectively suppresses thermal radiation in two atmospheric windows and enhances thermal radiation in the nonatmospheric window. Lu et al. proposed a magneto-controlled method to manipulate the transmittance properties of a graphene-based $\mathrm{THz}$ metasurface comprised of graphene cut-wire arrays. It is found that the introduced vertical electrostatic field deflects the carriers in graphene and changes the transmittance characteristics of the metasurface. Hu et al. demonstrated the anomalous launching and vortices generation of surface plasmons in a $\mathrm{THz}$ near-field metasurface platform by tuning the orientation as well as the geometric phase of the surface unit structure. By introducing nanofins made by phase change $\mathrm{VO}_{2}$ material into the metasurface, Song et al. proposed a temperaturecontrollable multifunctional metasurface lens based on phase transition material. The metasurface based lens can be switched among dual focus, single focus, and no focus at any position. For the similar focusing functionality, Yan et al. employed phase discontinuity of the three-layer square element based reflective metasurface for a high focusing efficiency of $82 \%$. Sun et al. further demonstrated a metasurface with three-layer aperture structures to achieve a beam deflection to the desired angle of high efficiency.

From the experimental side, various mechanisms for realizing tunable metasurface can be employed to create intelligent wave control devices. Wei et al. and Wei et al. proposed the 
switch transparency, reflection, and absorption of metasurfaces by loading PIN diodes in the structure. They experimentally demonstrated high power transmission modulation in the frequency range $8-12 \mathrm{GHz}$ and an active metasurface with a continuous changing absorption peak between 8.5 and $9.1 \mathrm{GHz}$. $\mathrm{Xu}$ et al. presented a review on the experimental progress of tunable metamaterials based on nematic liquid crystals. They showed that liquid crystal-based metamaterials are promising for the remarkable improvement of the bandwidth and may facilitate related applications at terahertz or even optical regimes.

This Research Topic provides an interesting overview of the different metamaterials and metasurfaces that incorporate tunable or reconfigurable mechanisms for breaking the limitation of

\section{REFERENCES}

1. Smith DR, Pendry JB, and Wiltshire MCK. Metamaterials and Negative Refractive Index. Science (2004) 305:788-92. doi:10.1126/science.1096796

2. Shalaev VM. Optical Negative-index Metamaterials. Nat Photon (2007) 1: 41-8. doi:10.1038/nphoton.2006.49

3. Soukoulis CM, and Wegener M. Past Achievements and Future Challenges in the Development of Three-Dimensional Photonic Metamaterials. Nat Photon (2011) 5:523-30. doi:10.1038/nphoton.2011.154

4. Yu N, Genevet P, Kats MA, Aieta F, Tetienne J-P, Capasso F, et al. Light Propagation with Phase Discontinuities: Generalized Laws of Reflection and Refraction. Science (2011) 334:333-7. doi:10.1126/science.1210713

5. Zheludev NI, and Kivshar YS. From Metamaterials to Metadevices. Nat Mater (2012) 11:917-24. doi:10.1038/nmat3431

6. Chen S, Liu W, Li Z, Cheng H, and Tian J. Metasurface-Empowered Optical Multiplexing and Multifunction. Adv Mater (2020) 32:1805912. doi:10.1002/ adma.201805912

7. Chen K, Ding G, Hu G, Jin Z, Zhao J, Feng Y, et al. Directional Janus Metasurface. Adv Mater (2020) 32:1906352. doi:10.1002/adma.201906352

8. Zhang F, Pu M, Li X, Gao P, Ma X, Luo J, et al. All-Dielectric Metasurfaces for Simultaneous Giant Circular Asymmetric Transmission and Wavefront Shaping Based on Asymmetric Photonic Spin-Orbit Interactions. Adv Funct Mater (2017) 27:1704295. doi:10.1002/adfm.201704295

9. Chen H-T, Yang H, Singh R, O'Hara JF, Azad AK, Trugman SA, et al. Tuning the Resonance in High-Temperature Superconducting Terahertz Metamaterials. Phys Rev Lett (2010) 105:247402. doi:10.1103/physrevlett.105.247402 narrow-operation wavelengths. These new results demonstrate recent progress in tunable metamaterials and their potential usefulness in various aspects, both experimentally and theoretically. We expect that more and more metamaterials/ metasurfaces will be demonstrated for fundamental wave control phenomena in physics and that novel functional metadevices with smart properties will soon emerge.

\section{AUTHOR CONTRIBUTIONS}

All authors listed have made a substantial, direct, and intellectual contribution to the work and approved it for publication.

10. Tassin P, Koschny T, and Soukoulis CM. Graphene for Terahertz Applications. Science (2013) 341:620-1. doi:10.1126/science.1242253

11. Fan Y, Shen N-H, Koschny T, and Soukoulis CM. Tunable Terahertz MetaSurface with Graphene Cut-Wires. ACS Photon (2015) 2:151-6. doi:10.1021/ ph500366z

12. Low T, and Avouris P. Graphene Plasmonics for Terahertz to Midinfrared Applications. ACS Nano (2014) 8:1086-101. doi:10.1021/ nn406627u

13. Ma M, Li Z, Liu W, Tang C, Li Z, Cheng H, et al. Optical Information Multiplexing with Nonlinear Coding Metasurfaces. Laser Photon Rev (2019) 13:1900045. doi:10.1002/lpor.201900045

14. Liu S, Cui TJ, Xu Q, Bao D, Du L, Wan X, et al. Anisotropic Coding Metamaterials and Their Powerful Manipulation of Differently Polarized Terahertz Waves. Light Sci Appl (2016) 5:e16076. doi:10.1038/ lsa.2016.76

Conflict of Interest: The authors declare that the research was conducted in the absence of any commercial or financial relationships that could be construed as a potential conflict of interest.

Copyright (c) 2021 Fan, Zhao, Zhang and Shen. This is an open-access article distributed under the terms of the Creative Commons Attribution License (CC BY). The use, distribution or reproduction in other forums is permitted, provided the original author(s) and the copyright owner(s) are credited and that the original publication in this journal is cited, in accordance with accepted academic practice. No use, distribution or reproduction is permitted which does not comply with these terms. 